\title{
Standardized uptake value variations of normal glandular breast tissue at dual time point FDG-PET/CT imaging
}

\author{
Ashraf Anas Zytoon \\ Radiology Department, Faculty of Medicine, Menoufiya University, PET Center, Dokkyo Medical University, Japan \\ Email address: \\ ashradio@gmail.com
}

To cite this article:

Ashraf Anas Zytoon. Standardized Uptake Value Variations of Normal Glandular Breast Tissue at Dual Time Point FDG-PET/CT Imaging. International Journal of Medical Imaging. Vol. 1, No. 3, 2013, pp. 56-65. doi: 10.11648/j.ijmi.20130103.14

\begin{abstract}
Purpose: To study the relationship between (FDG) uptake expressed quantitatively as standardized uptake values (SUVs) in normal breast tissues and breast density, age, menopausal status determined during dual-time point FDGPET/CT imaging. Materials and Methods: maximum and average SUVs were determined in two hundred eighty four patients (all females; mean age 55.5 \pm 14.1 range 13-84 years, 115 premenopausal, 169 postmenopausal) with newly diagnosed unilateral breast cancer, gynecological cancer and lung cancer were analyzed. One hundred forty two (50\%) patients had dense breast whereas $142(50 \%)$ patients had non-dense breast according to the ACR Lexicon criteria. All the patients underwent dual time point imaging sequential PET/CT scans for preoperative staging. In the present study, we analyzed maximum and average SUVs for the normal breast parenchyma and nipple regions. Results: Of the 284 normal breast parenchyma; $64.4 \%$ showed a decrease and $35.6 \%$ showed either no change $20.1 \%$ or an increase $15.5 \%$ in SUV over time. Similar values for the normal tissue in the nipple region were; $77.5 \%$ showed a decrease and $22.5 \%$ showed either no change $13 \%$ or an increase $9.5 \%$ in SUV over time. There was significant difference in maximum and average SUVs of breast parenchyma and nipple in patients with dense and nondense breasts $(p<0.0001)$. There were trends of negative relationship between physiological FDG uptake and age $(p<0.0001)$. Furthermore, there was significant difference in maximum and average SUVs of breast parenchyma with different menopausal status $(p<0.0001)$. Our analysis revealed that breast density, age and menopausal status were significant predictors for FDG uptake in the normal breasts. Conclusion: There was a significant difference in SUVs between the dense and non-dense normal breast. Menopausal status and age do significantly affect the uptake of FDG. Delayed phase imaging can improve the accuracy of the test in the evaluation of breast cancer as the physiological FDG uptake decrease so the pathological uptake becomes prominent and could be easily depicted.
\end{abstract}

Keywords: Breast Cancer, FDG-PET/CT, Standardized Uptake Value (SUV), Dual-Time Point Imaging, Delayed Imaging, Breast Density, Menopause

\section{Introduction}

Breast cancer is the commonest cancer in women and is the second leading cause of cancer deaths [1]. Although it is curable when detected early, about one third of women with breast cancer die of the disease [2]. Screening with conventional mammography along with physical examination is a sensitive method for the early detection of breast cancer and has also been shown to decrease associated mortality [3-7].

Mammography reduces breast cancer mortality [5] but, especially in women with dense breasts, has limited diagnostic accuracy, which results in a considerable number of missed cancers. Breast density is a measure of stromal and epithelial breast tissue [8] and is classified into 1 of 4 groups as defined by the Breast Imaging Reporting And Data System (BI-RADS): almost entirely fatty (group 1), scattered fibroglandular tissue (group 2), heterogeneously dense (group 3), and extremely dense (group 4). Moreover, the sensitivity of mammography for detecting breast cancer declines significantly with increasing breast density [9-10].

Mammography detects breast cancer with sensitivities ranging from only $30 \%$ to $68 \%$ in women with dense or very dense breasts. In women aged 50-64 y who had dense breasts and had undergone estrogen replacement, the sensitivity was 55\%. [3, 10-11]. In addition, diagnosis can be difficult in young women with dense breasts, in those with implants, and after surgery or irradiation to the breast 
tissue [12].

Mandelson et al. [10] reported sensitivities for breast cancer detection of $80 \%, 59 \%$, and $30 \%$ in women with predominantly fatty breast tissue, heterogeneously dense breasts, and extremely dense breasts, respectively. The authors concluded that "breast density is one of the strongest, if not the strongest, predictor of the failure of mammographic screening to detect cancer". Finally, increasing mammographic density is associated with a higher false-positive rate [13]. Foxcroft et al. [11] reported similar limitations for mammographic screening and recommended that women with dense breasts should undergo different screening tests.

2-Deoxy-2-[F-18] fluoro-D-glucose (FDG)-positron emission tomography (PET) imaging has been proposed as a diagnostic modality for improved detection of breast cancer and is shown to be highly accurate in characterizing palpable breast lesions [14-16]. One of the important advantages of FDG-PET over other imaging techniques is that it can provide a very high measure of contrast between normal and malignant tissues. FDG-PET has been well established in differentiating between benign and malignant tumors including breast cancer and is also of value in evaluating loco-regional spread and distant metastasis [17-18].

Similar to mammography, breast density may also have an effect on the interpretation of FDG-PET. This was confirmed for the first time in a retrospective study by Vranjesevic et al. [19]. Because dense breasts contain more cells per volume, one may expect an overall higher uptake of FDG in these tissues, which could make the diagnosis of breast malignancies more difficult. In our own experience, we have noticed that patients with clinically dense breasts tend to have increased FDG uptake on PET [20].

It has been reported, that the accuracy of FDG-PET/CT could be further enhanced by dual-time point imaging. However, this method is not universally used. The present study was aimed at assessing the pattern of physiological uptake by normal breast tissue expressed by standardized uptake value (SUV) at both phases of dual time point FDG-PET/CT imaging in normal breast with variations of age, breast density, and hormonal status.

\section{Patients and Methods}

\subsection{Patient Population}

This prospective study was approved by the review board of our institute. Written informed consent was obtained from all patients. Two hundred eighty four patients (all females; age mean $55.5 \pm 14.1,95 \%$ CI 53.957.2, median 57, range 13-84 years, premenopausal 115; postmenopausal 169) with newly diagnosed unilateral breast cancer, gynecological cancer (ovarian cancer, cervical cancer, endometrial cancer, benign leiomyoma), and lung cancer were analyzed. In the present study, we retrospectively analyzed maximum and average SUVs for the normal breast (142 right and 142 left breast). One hundred forty two $(50 \%)$ patients had grade III or IV mammographic density (dense breast) whereas $142(50 \%)$ patients had grade I or II breast density (nondense breast) according to the ACR Lexicon criteria. The breast tissue density was suggested by mammography. All the mammographic studies were obtained within 4 weeks before to the FDG-PET scan. The patients underwent multimodality imaging techniques, such as digital mammography, ultrasonography, CT, MRI, and FDGPET/CT. None of the patients had received chemotherapy or radiation therapy before they underwent dual time point FDG-PET/CT for preoperative staging.

Table 1. Basic Clinical and Pathological Characteristics of the Patients

\begin{tabular}{|c|c|}
\hline No. of Patients & 284 \\
\hline Sex & all female \\
\hline $\begin{array}{l}\text { Age (years); mean }(95 \% \mathrm{CI} \text {, } \\
\text { median, range) }\end{array}$ & $55.5 \pm 14.1(53.9-57.2,57,13-84)$ \\
\hline \multicolumn{2}{|l|}{ Histopathology } \\
\hline Unilateral breast cancer & 164 \\
\hline Lung cancer & 60 \\
\hline Gynecological cancer & 60 \\
\hline \multicolumn{2}{|c|}{ SUVmax normal breast; mean ( $95 \% \mathrm{CI}$, median, range) } \\
\hline SUVmax 1 & $1.11 \pm 0.47(1.06-1.17,1.10,0.50-3.60)$ \\
\hline SUVmax2 & $0.94 \pm 0.47(0.88-0.99,0.90,0.20-3.20)$ \\
\hline$\Delta \mathrm{SUVmax} \%$ & $\begin{array}{l}-13.24 \pm 25.90(-16.35--10.13,-18.20,- \\
80.00-100.00)\end{array}$ \\
\hline \multicolumn{2}{|c|}{ SUVmax normal nipple; mean (95\% CI, median, range) } \\
\hline SUVmax1 & $1.18 \pm 0.40(1.11-1.24,1.10,0.50-2.20)$ \\
\hline SUVmax2 & $0.86 \pm 0.34(0.80-0.92,0.80,0.20-1.70)$ \\
\hline$\triangle \mathrm{SUVmax} \%$ & $\begin{array}{l}-26.20 \pm 26.70(-31.30--21.10,-33.30,- \\
60.00-60.00)\end{array}$ \\
\hline \multicolumn{2}{|c|}{ SUVav normal breast; mean ( $95 \%$ CI, median, range) } \\
\hline SUVav1 & $0.52 \pm 0.23(0.48-0.56,0.50,0.20-1.30)$ \\
\hline SUVav2 & $0.40 \pm 0.16(0.37-0.43,0.40,0.10-0.70)$ \\
\hline$\Delta \mathrm{SUVav} \%$ & $\begin{array}{l}-15.80 \pm 38.49(-22.85--8.75,-25.00,- \\
66.70-133.30\end{array}$ \\
\hline \multicolumn{2}{|c|}{ SUVav normal nipple; mean (95\% CI, median, range) } \\
\hline SUVav1 & $0.58 \pm 0.20(0.54-0.61,0.60,0.20-1.10)$ \\
\hline SUVav2 & $0.45 \pm 0.18(0.41-0.48,0.45,0.10-0.80)$ \\
\hline$\Delta \mathrm{SUVav} \%$ & $\begin{array}{l}-19.00 \pm 39.53(-26.52--11.44,-22.5,- \\
66.70-150)\end{array}$ \\
\hline
\end{tabular}

\subsection{FDG-PET/CT Imaging and Assessment}

Patients fasted for at least $6 \mathrm{~h}$ before the PET scan and had blood glucose levels of $<140 \mathrm{mg} / \mathrm{dl}$ at the time of injection. PET/CT was performed with a dedicated wholebody PET scanner (Biograph Sensation 16 PET-CT Scanner; Siemens Medical Systems). All the patients underwent dual 
time point imaging sequential PET/CT scans for preoperative staging with an average time interval of approximately 50-60 min between the two phases. The first scan was performed as whole-body images from head to thigh, with acquisition of 6-7 bed positions; resulting in a complete axial length of $80-100 \mathrm{~cm}$. Imaging began 55-60 min after injection of ${ }^{18} \mathrm{~F}-\mathrm{FDG}(4.5 \mathrm{MBq} / \mathrm{kg}$ of body weight). In all cases, ${ }^{18} \mathrm{~F}$-FDG radiochemical purity was $>$ $95 \%$ and specific activity was $>47 \mathrm{GBq} / \mu \mathrm{mol}$. FDG was infused using a volume of 8-15 ml over 2-3 minutes in the antecubital vein contralateral to the affected breast. Delayed imaging began at approximately 110-120 min after FDG injection. Transmission scans were performed for all patients to provide attenuation correction with CT. The PET slice thickness was $3.4 \mathrm{~mm}$. Three-dimensional (3D) data were acquired without septa, with image reconstruction and scatter correction using a computer system (e-soft nuclear medicine acquisition, processing, and viewing software). Visual assessment included assessing both sets of images at the same time. Analysis was based on measuring the maximum and average FDG uptake at the normal breast at both time points.

\subsection{Image Analysis}

PET images were reconstructed using measured attenuation correction, dead-time correction and decay correction to the beginning of each scan. The maximum (SUVmax) and average (SUVav) standardized uptake value of FDG was measured from both time points; time point 1 (SUV1) and time point 2 (SUV2) for the breast parenchyma proper and nipple region. Visual assessment, image interpretation and data analysis were performed independently by two expert dual qualified radiology/nuclear medicine physicians. They were aware of the patients' clinical history, which was provided by the referring physician, but were blinded to the results of other imaging studies if these were performed. When there was a difference between the two observers, a mean was calculated to determine the final SUV. After image reconstruction, a free-hand ROI was carefully overlaid onto 3-6 PET scan slices at the site of the normal breast tissue. The SUVs for imaging at the second time point were obtained with the same techniques as those used for imaging at the first time point. The maximum and average FDG uptake on the consecutive early scans was obtained for quantitative measurement of the metabolic activity of the tracer. From the ROIs, the SUV was calculated according to the following formula: (mean ROI activity $[\mathrm{MBq} / \mathrm{g}]) /($ injected dose $[\mathrm{MBq}] /$ body weight $[\mathrm{g}])$, where $\mathrm{g}$ $=$ grams. The $\Delta \mathrm{SUV} \%$ change over time (retention index RI) is defined as follows: (SUV2-SUV1)/(SUV1)x100, where SUV1 and SUV2 are the values of SUV at the initial and delayed phases, respectively. $\triangle \mathrm{SUV} \%$ is usually expressed as a percentage. The percentage change in the SUV of the normal breast between the 2 time points was calculated for the purpose of the investigation.

Previously reported that there is no significant difference was noted in maximum and average SUV among right and left breasts as well as the right and left nipple (20). The PET results from different semiquantitative parameters (SUVmax, SUVav) were then compared together between different groups to estimate the optimal method with the highest accuracy for estimating the physiological uptake at the normal breast tissue for the purpose of differentiating physiological from pathological uptake.

\subsection{Statistical Analysis}

Descriptive statistics were used to summarize the baseline clinical profile/data. Paired t test was used to obtain the mean significant difference among values of uptake between the left and right breasts, whereas unpaired $t$ test was applied to assess the mean significant difference among the uptake between premenopausal and postmenopausal, and dense and nondense breasts. One-way analysis of variance (ANOVA) with post-hoc analysis was used to determine the mean significant difference among the values of uptake in various categories of age ( $\leq 44$ years, $45-55,56-65$ and $\geq 66$ years).

Bivariate Pearson correlation was used to examine the linear relationship between various quantitative variables. In addition, stepwise multiple regression analysis was carried out to assess the effect of several independent covariates such as age, breast density, menopausal status, etc., on dependent (outcome) variable uptake. A p value of less than 0.05 was considered as a significant level.

To analyze the relationship between age, menopause state, breast tissue density and SUV; patients were sorted into groups according to age patients were divided into four groups (I: $\leq 44$ years, II: $45-55$ years, III: $56-65$ years and IV: $\geq 66$ years), according to menoupause state into two groups (premenopausal and post menopausal) and according to breast tissue histological features, patients were divided dense and nondense breast. All semiquantitative data were expressed in terms of mean \pm SD. All statistical analyses were two sided with significance defined as $P<0.05$. Three levels of statistics were performed. Analysis of the correlationship between early and delayed phases parameters was performed by Spearman's rank test to determine the correlation coefficient of the semiquantitative parameters change overtime between the early and delayed phases.

\section{Results}

Two hundred and eighty four female patients were investigated in this study. Patient characteristics are age; mean 55.5 $\pm 14.1,95 \%$ CI 53.9-57.2, median 57, range 1384 years, menopausal status; 115 premenopausal, 169 postmenopausal, 142 right and 142 left breast. They present with newly diagnosed unilateral breast cancer, gynecological cancer (ovarian cancer, cervical cancer, endometrial cancer, benign leiomyoma), and lung cancer. One hundred forty two $(50 \%)$ patients had grade III or IV mammographic density (dense breast) whereas 142 (50\%) 
patients had grade I or II breast density (nondense breast) according to the ACR Lexicon criteria.

\subsection{SUV Measurements}

In all patients, the SUVmax and SUVav could be calculated for the normal breast parenchyma mammary tissue proper and nipple area. Of the 284 normal breast parenchyma; $183(64.4 \%)$ showed a decrease and 101 $(35.6 \%)$ showed either no change $\{57(20.1 \%)\}$ or an increase $\{44(15.5 \%)\}$ in SUV over time (figure 1.1.). Similar values for the normal tissue in the nipple region were; $220(77.5 \%)$ showed a decrease and $64(22.5 \%)$ showed either no change $\{37(13 \%)\}$ or an increase $\{27$ $(9.5 \%)\}$ in SUV over time (figure 1.2.).

\section{Normal Breast Parenchyma Physiological Uptake Patterns}

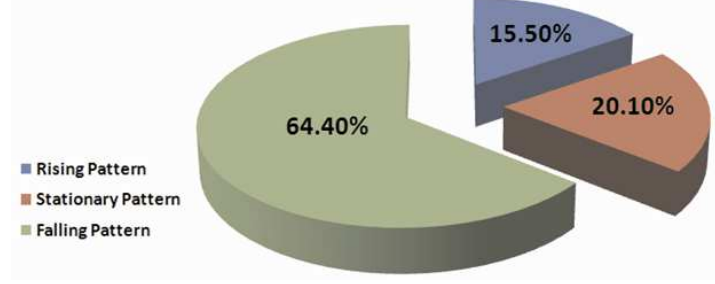

Figure 1.1.

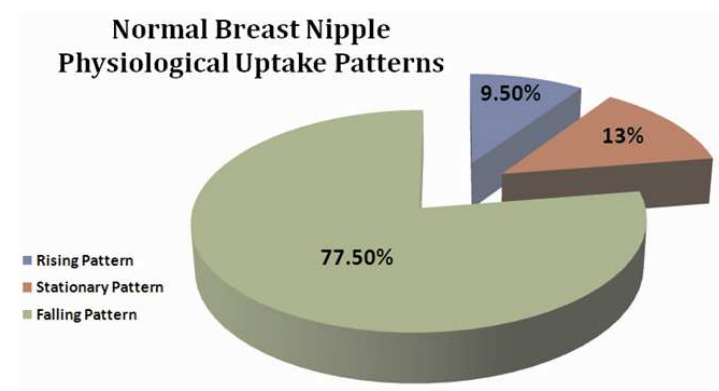

Figure 1.2.

Normal breasts parenchyma maximum standardized uptake values (SUVmax); SUVmax1, SUVmax2, and $\triangle \mathrm{SUVmax} \%$ mean values were; $1.11 \pm 0.47,0.94 \pm 0.47$, and $-13.24 \pm 25.90$ while average standardized uptake values (SUVav); SUVav1, SUVav2, and $\triangle \mathrm{SUVav} \%$ mean values were; $0.52 \pm 0.23,0.40 \pm 0.16$, and $-15.80 \pm 38.49$. Similar values for the normal tissue in the nipple region were $1.18 \pm 0.40,0.86 \pm 0.34$, and $-26.20 \pm 26.70$ for SUVmax values, and $0.58 \pm 0.20,0.45 \pm 0.18$, and $-19.00 \pm$ 39.53 for SUVav values.

The highest maximum SUV and average SUV at the early phase and delayed phases for the normal breast parenchyma were $3.60,1.30$ for the early phase and 3.20 , 0.70 for the delayed phase respectively. The same values for the nipple were $2.20,1.10$ for the early phase and 1.70 , 0.80 for the delayed phase respectively. There was no significant difference in SUVs between breast parenchyma and nipple $(P=0.2607,0.0828$ for the early and delayed phases respectively).

The percentage change (decrease) between SUVmax 1 and SUVmax2 as well as between SUVav1 and SUVav2 over time was significant $(P<0.0001)$ (figures 2.1., 2.2.). (figures 3.1., 3.2.) are before-after plot graphs displaying change (decrease) in nipple region SUVmax and SUVav against time interval between the dual scans. It demonstrates a significant correlation between SUVmax and SUVav change and time interval $(P<0.0001)$.

Of the 44 normal breasts that showed an increase in SUV over time, there were no obvious focal lesions in the follow up imaging. While breast cancer SUVmax increased over time by $19.1 \%$ [21], the mean physiological uptake of normal breast tissue parenchyma and nipple region decreased over time by $-13.24 \%,-26.20 \%$ for maximum $(\Delta$ SUVmax $\%)$ and $-15.80,-19.00 \%$ for average ( $\triangle$ SUVav $\%$ ) uptake values.

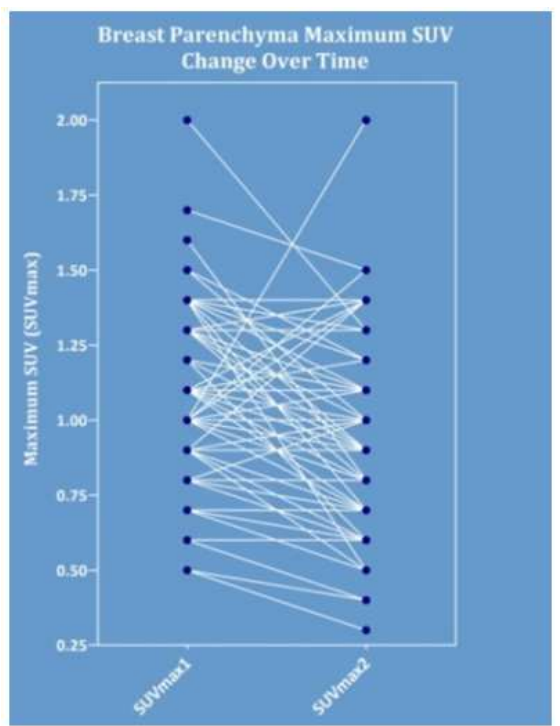

Figure 2.1. Breast Parenchyma Maximum SUV Change Over Time

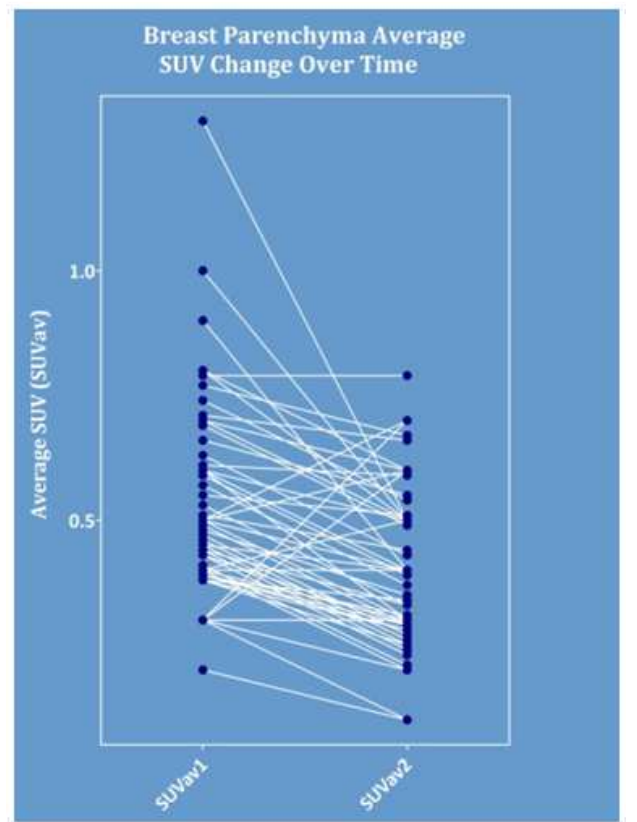

Figure 2.2. Breast Parenchyma Average SUV Change Over Time 


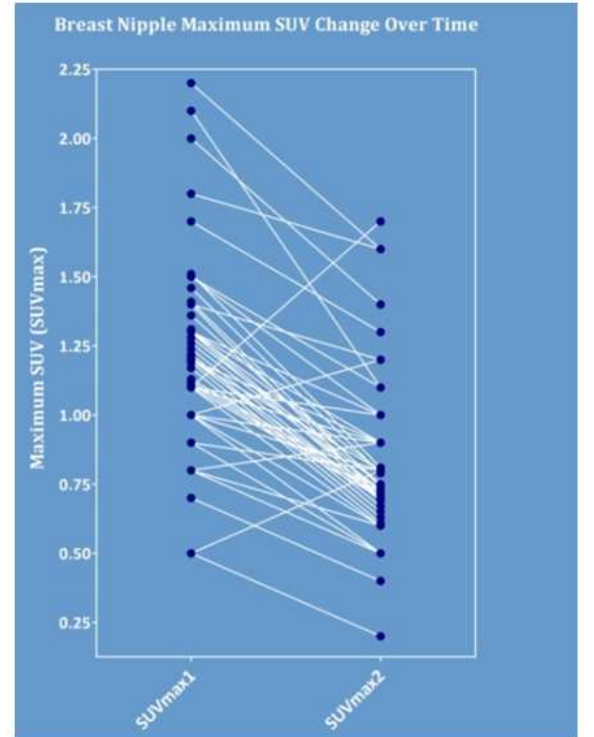

Figure 3.1. Breast Nipple Maximum SUV Change Over Time

\subsection{Relationship of SUV with Breast Density}

Maximum and average SUVs for normal tissue of dense breasts were $1.32 \pm 0.52$ and $0.72 \pm 0.42$ at the early phase, $1.20 \pm 0.53$ and $0.52 \pm 0.11$ at the delayed phase and -8.56 \pm 32.21 and $-1.06 \pm 50.50$ for the change over time $(\Delta \mathrm{SUVmax} \%, \Delta \mathrm{SUVav} \%)$ between the early and delayed phases respectively. Similar values for the non dense breasts were $0.88 \pm 0.22$ and $0.41 \pm 0.12$ at the early phase,

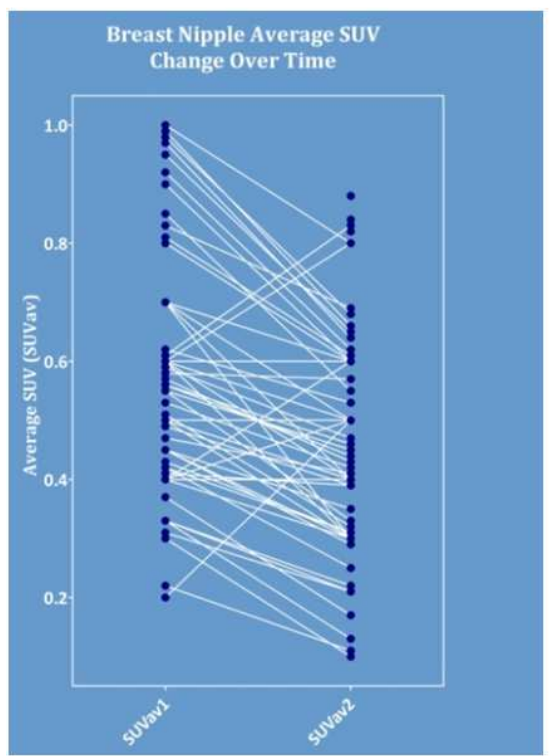

Figure 3.2. Breast Nipple Average SUV Change Over Time

$0.71 \pm 0.23$ and $0.27 \pm 0.11$ at the delayed phase, $-8.67 \pm$ 44.30 and $-29.51 \pm 21.67$ for the SUV change over time $(\triangle \mathrm{SUVmax} \%, \Delta \mathrm{SUVav} \%)$ between the early and delayed phases respectively (table 2). There was significant difference in maximum and average SUVs of breast parenchyma in patients with dense and nondense breasts $(p$ $<0.0001$; figures 4.1., 4.2.).

Table 2. Showing the mean values of SUVs (early phase, delayed phase) in patients with different breast densities

\begin{tabular}{lccccccccc}
\hline $\begin{array}{l}\text { Menopausal } \\
\text { Status }\end{array}$ & $\begin{array}{c}\text { No. of } \\
\text { patients }\end{array}$ & Maximum SUV (breast) & \multicolumn{2}{c}{ Average SUV (breast) } & \multicolumn{2}{c}{ Maximum SUV (nipple) } \\
\multicolumn{1}{c}{ EARLY } & DELAYED & EARLY & DELAYED & EARLY & DELAYED & EARLY & DELAYED \\
\hline $\begin{array}{l}\text { Dense Breast } \\
\begin{array}{l}\text { Non Dense } \\
\text { Breast }\end{array}\end{array}$ & 142 & $1.32 \pm 0.52$ & $1.20 \pm 0.53$ & $0.72 \pm 0.42$ & $0.52 \pm 0.11$ & $1.36 \pm 0.40$ & $0.96 \pm 0.30$ & $0.68 \pm 0.20$ & $0.53 \pm 0.16$ \\
\hline
\end{tabular}

Maximum and average SUVs for nipple of dense breasts were $1.36 \pm 0.40$ and $0.68 \pm 0.20$ at the early phase, $0.96 \pm$ 0.30 and $0.53 \pm 0.16$ at the delayed phase and $-30.09 \pm$ 12.30 and $-20.19 \pm 27.84$ for the change over time $(\triangle \mathrm{SUVmax} \%, \Delta \mathrm{SUVav} \%)$ between the early and delayed phases respectively. Similarly, the values for the nondense breasts were $0.99 \pm 0.28$ and $0.49 \pm 0.15$ at the early phase, $0.76 \pm 0.33$ and $0.38 \pm 0.17$ at the delayed phase, $-21.77 \pm$ 33.53 and $-20.03 \pm 47.50$ for the SUV change over time ( $\triangle \mathrm{SUVmax} \%, \Delta \mathrm{SUVav} \%$ ) between the early and delayed phases respectively (table 2). There was significant difference in SUVs of nipple in patients with dense and nondense breasts ( $p<0.0001$; figures 5.1., 5.2.).

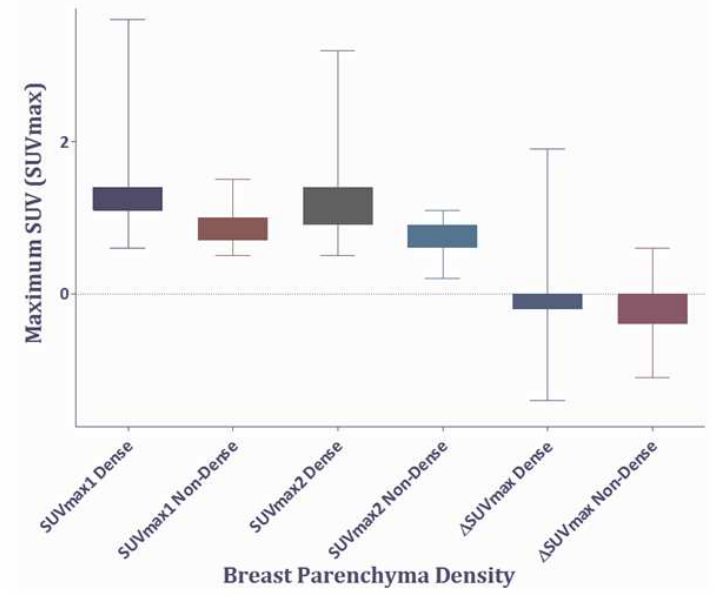

Figure 4.1. Relationship between maximum SUV and parenchymal breast density. 


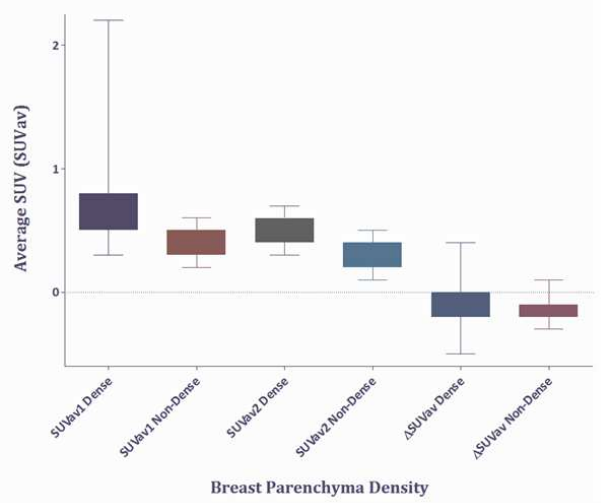

Figure 4.2. Relationship between average SUV and parenchymal breast density.

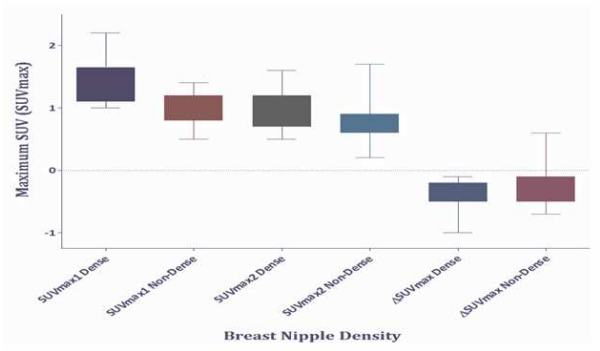

Figure 5.1. Relationship between maximum SUV and nipple breast density.

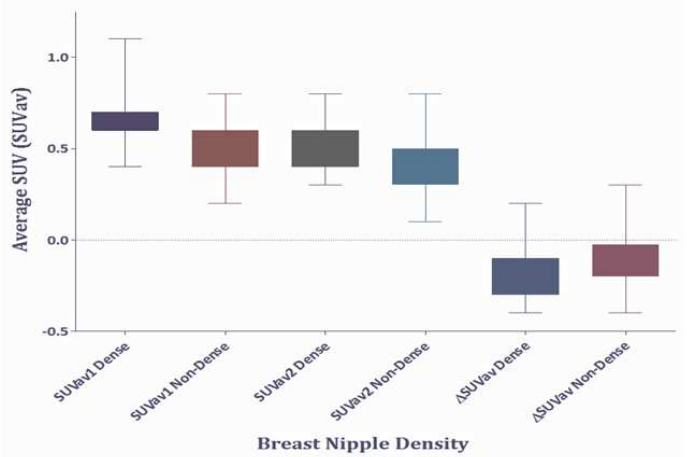

Figure 5.2. Relationship between average SUV and nipple breast density.

The relationship of SUVs with breast density is shown in graphs (figures 4 and 5). No significant difference was noted in maximum and average SUVs among right and left breasts as well as right and left nipple. Typical breast PET, $\mathrm{CT}$ and corresponding PET/CT slices of dense (figures 6AC) and non-dense (figures 6D-F) categories are given in figure 6.

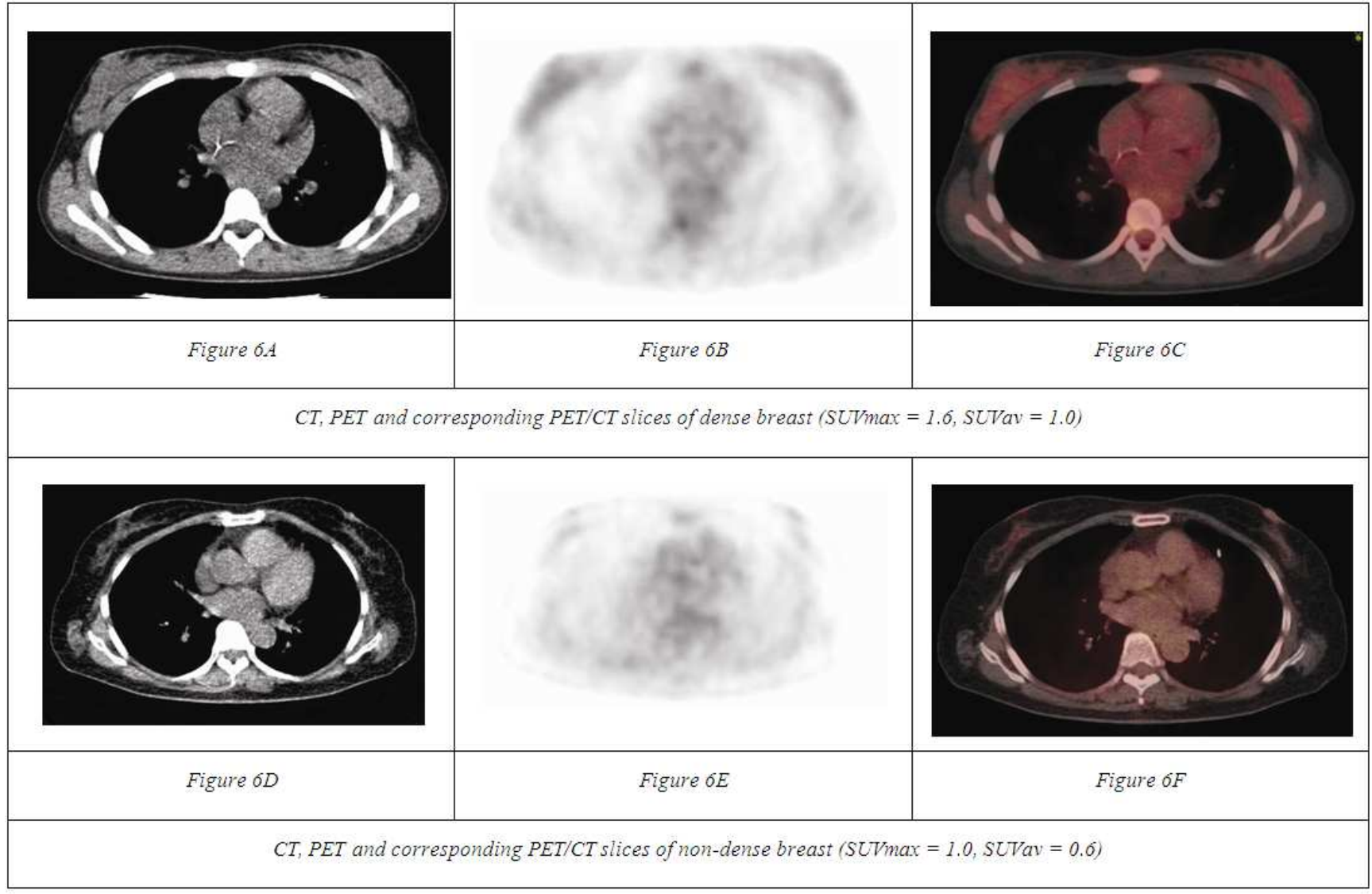

\subsection{Relationship of SUV with Age}

The mean values of maximum and average SUVs for breast parenchyma and nipple in four different age groups are given in table 3 . 
Table 3. Showing the mean values of SUVS (early phase, delayed phase) in patients with different age groups

\begin{tabular}{llllllllll}
\hline \multirow{2}{*}{ Age (years) } & $\begin{array}{l}\text { No. of } \\
\text { patients }\end{array}$ & \multicolumn{2}{l}{ Maximum SUV (breast) } & \multicolumn{2}{c}{ Average SUV (breast) } & \multicolumn{2}{c}{ Maximum SUV (nipple) } & \multicolumn{2}{c}{ Average SUV (nipple) } \\
& EARL & DELAYED & EARLY & DELAYED & EARLY & DELAYED & EARLY & DELAYED \\
\hline$\leq 44$ years & 61 & $1.48 \pm 0.77$ & $1.28 \pm 0.77$ & $0.86 \pm 0.54$ & $0.55 \pm 0.10$ & $1.17 \pm 0.40$ & $1.02 \pm 0.39$ & $0.77 \pm 0.19$ & $0.57 \pm 0.19$ \\
45-55 years & 75 & $1.14 \pm 0.31$ & $0.91 \pm 0.31$ & $0.44 \pm 0.11$ & $0.36 \pm 0.16$ & $1.28 \pm 0.44$ & $0.89 \pm 0.34$ & $0.61 \pm 0.20$ & $0.48 \pm 0.22$ \\
$56-65$ years & 72 & $1.05 \pm 0.24$ & $0.98 \pm 0.35$ & $0.54 \pm 0.15$ & $0.43 \pm 0.10$ & $0.99 \pm 0.22$ & $0.83 \pm 0.29$ & $0.48 \pm 0.14$ & $0.42 \pm 0.13$ \\
$\geq 66$ years & 76 & $0.86 \pm 0.22$ & $0.70 \pm 0.26$ & $0.38 \pm 0.18$ & $0.28 \pm 0.18$ & $1.06 \pm 0.33$ & $0.70 \pm 0.31$ & $0.51 \pm 0.13$ & $0.36 \pm 0.12$ \\
\hline
\end{tabular}

There was significant difference in maximum and average SUVs of breast parenchyma and nipple in patients of different age groups $(p<0.0001)$. However, there were trends of negative relationship, i.e., decreasing SUVs with increasing age (figures 7.1., 7.2., 8.1., 8.2.).

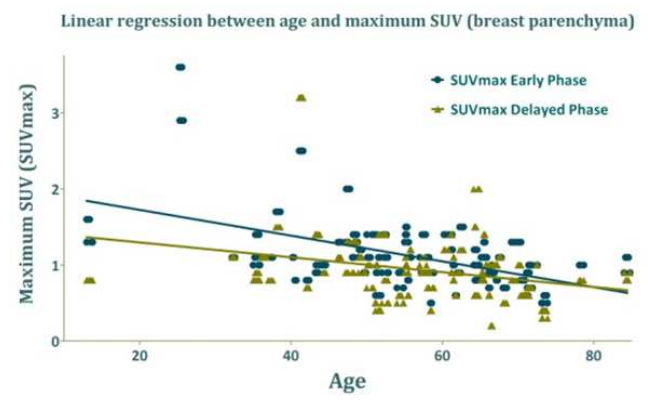

Figure 7.1. $R=-0.47(95 \% C I-0.56$ to $-0.37, p<0.0001)$

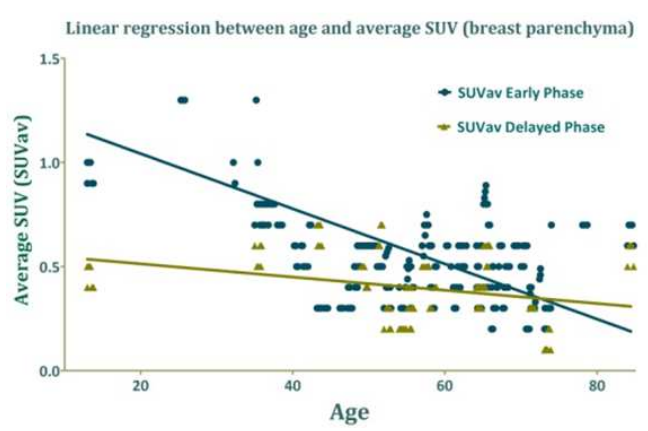

Figure 7.2. $R=-0.39(95 \% C I-0.48$ to $-0.28, p<0.0001)$

\subsection{Relationship of SUV with Menopausal Status}

The mean values of maximum and the average SUVs for right breast, left breast, right nipple, and left nipple in patients with premenopausal, perimenopausal, and postmenopausal status are given in table 4 . There was significant difference in maximum and average SUVs of

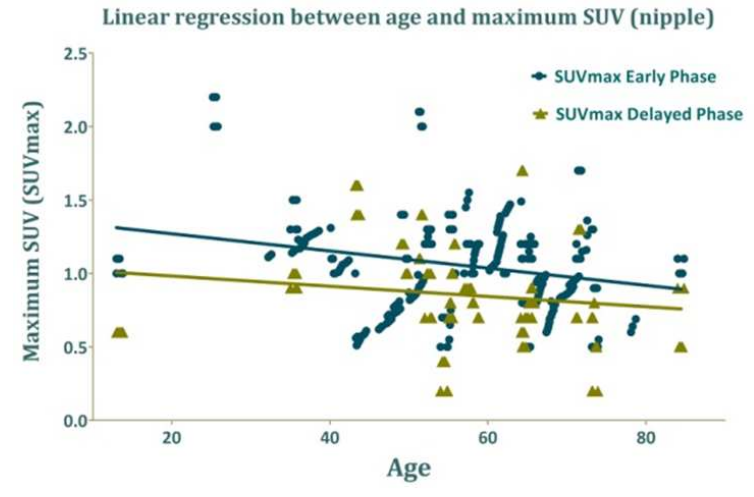

Figure 8.1. $R=-0.20(95 \% C I-0.31$ to $-0.08, p=0.0009)$

Linear regression between age and average SUV (nipple)

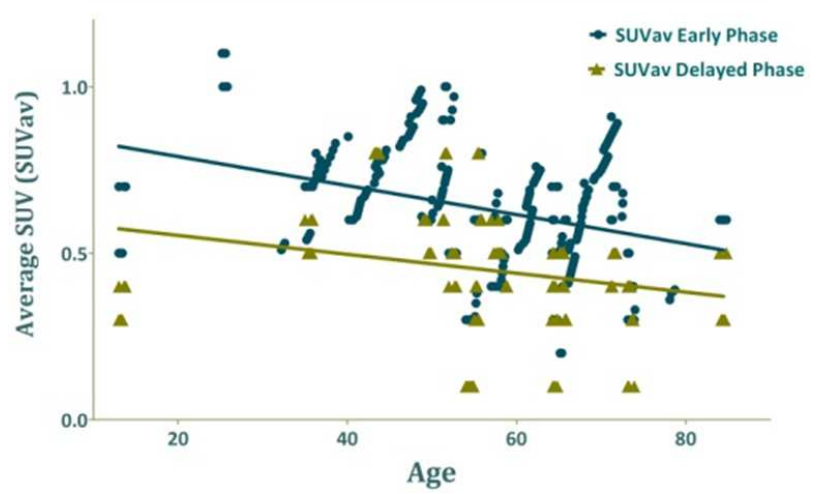

Figure 8.2. $R=-0.35(95 \% C I-0.45$ to $-0.24, p<0.0001)$

breast parenchyma and nipple in patients with different menopausal status $(p<0.0001)$.

Our analysis revealed that breast density, age and menopausal status were significant predictors for FDG uptake in the normal breasts.

Table 4. Showing the mean values of SUVS (early phase, delayed phase) in patients with different menopausal status

\begin{tabular}{|c|c|c|c|c|c|c|c|c|c|}
\hline \multirow{2}{*}{$\begin{array}{l}\text { Menopausal } \\
\text { Status }\end{array}$} & \multirow{2}{*}{$\begin{array}{l}\text { No. of } \\
\text { patients }\end{array}$} & \multicolumn{2}{|c|}{ Maximum SUV (breast) } & \multicolumn{2}{|c|}{ Average SUV (breast) } & \multicolumn{2}{|c|}{ Maximum SUV (nipple) } & \multicolumn{2}{|c|}{ Average SUV (nipple) } \\
\hline & & EARLY & DELAYED & EARLY & DELAYED & EARLY & DELAYED & EARLY & DELAYED \\
\hline Premenopausal & 115 & $1.33 \pm 0.62$ & $1.10 \pm 0.60$ & $0.72 \pm 0.47$ & $0.50 \pm 0.15$ & $1.45 \pm 0.43$ & $1.04 \pm 0.31$ & $0.71 \pm 0.20$ & $0.57 \pm 0.16$ \\
\hline Postmenopausal & 169 & $0.97 \pm 0.26$ & $0.84 \pm 0.32$ & $0.46 \pm 0.16$ & $0.35 \pm 0.15$ & $1.02 \pm 0.29$ & $0.77 \pm 0.31$ & $0.50 \pm 0.15$ & $0.39 \pm 0.17$ \\
\hline
\end{tabular}




\section{Discussion}

Conventional screen-film mammography has limited sensitivity for detection of breast cancer especially in breasts with dense fibroglandular tissue [9]. Digital mammography was developed to address some of the limitations of screen film mammography. However, accuracy of digital mammography is not substantially different from that of screen film mammography [22]. FDG-PET has been shown to be highly useful for management of breast cancer [23].

To our knowledge, this was the first study to examine the impact of breast density, age and hormonal status, on FDG uptake in normal breast tissue during dual time point PET/CT imaging. The study revealed that FDG uptake in normal breast tissue, as expressed by the SUV, is affected by breast density, and patient age. Menopausal status also had a significant impact on SUV, but to a lesser degree.

The metabolic activity of normal dense breast tissue was low; the mean SUVmax encountered was 1.32 and 1.20 for the breast parenchyma, and 1.36 and 0.96 for the nipple region at the early and delayed phases respectively (table 1). Thus, the mean SUVmax was well below the threshold value of 2.5-3.5 that is frequently used as a cutoff point for discriminating benign from malignant lesions. Thus, in spite of breast density could affect FDG uptake, it could not affect breast cancer delectability, besides delayed phase scan seems essential in such confusing patients where the physiological FDG uptake decrease and pathological FDG uptake increase [24].

Avril et al. [17] have systematically analyzed FDG uptake in benign and malignant primary breast lesions. In malignant tumors, a mean SUV of $3.3 \pm 1.8$ was reported, whereas in benign breast tumors, the mean SUV was $1.4 \pm$ 0.5 . This difference in tumor SUVs was statistically significant $(P<0.001)$.

In the current study, maximum and average FDG uptake was not significantly different in the right breast than in the left breast. The statistical analysis revealed nonsignificant left to right asymmetries for the different breastdensity groups.

Because the prevalence of mammographically dense breasts declines with age and dense breast tissue is more common before than after menopause, it has been suggested that menopausal status, rather than age, is the most important determinant of breast density [25]. The current study found significant correlation between age, menopausal status and FDG uptake.

Increased breast density is considered an independent risk factor for developing breast cancer. Recent studies reported the impact of breast density on FDG uptake in women with normal breast tissue. Vranjesevic et al. [19] retrospectively studied 45 women who had undergone whole body FDG-PET for indications other than breast cancer. In their study, Kumar et al. [20] study concluded that breast density and menopausal status affect the uptake of FDG, with average and peak SUVs significantly higher in dense versus fatty breasts $(p=0.01)$. That preliminary results also revealed that the maximum and the average SUVs of normal dense breasts were significantly higher than those of normal nondense breasts ( $p=0.003$ for both).

In a recent study evaluated the relationship of SUVs in normal breast with age, breast density, and menopausal status. Analysis of data of 96 patients showed that normal tissue of dense breasts had significantly higher maximum and average uptake of FDG compared to nondense breasts $(p<0.001$ for both) [26]. Compared to the results gathered by Vranjesevic et al. [19], in their study Kumar et al. report slightly higher peak and average SUVs in both dense and nondense breasts. Such higher SUVs are most likely attributable to the difference in defining the ROI between the two studies. The mean of maximum SUVs for both dense and nondense breast was 1.02 and 0.66 for breast parenchyma, and 0.85 and 0.76 for the nipple, respectively. In the current study the highest maximum SUV and average SUV at the early phase and delayed phases for the normal breast parenchyma were $3.60,1.30$ for the early phase and $3.20,0.70$ for the delayed phase respectively. The same values for the nipple were $2.20,1.10$ for the early phase and $1.70,0.80$ for the delayed phase respectively. There was no significant difference in SUVs between breast parenchyma and nipple $(P=0.2607,0.0828$ for the early and delayed phases respectively).

These higher SUVs in dense breasts are more attributable to fibrogladular tissue in dense breasts as compared to nondense breasts. The highest maximum SUVs for all normal breast tissues were below 3.6 at the early phase and 3.2 at the delayed phased for breast parenchyma and $\leq 2.2$, 1.7 for nipple region. These maximum SUVs of breast parenchyma and nipple are considerable lower in the delayed phase than in the early phase so it would be to depict slowly growing malignancy with low-avid FDG uptake pattern and small tumors in the delayed phase.

Our results show that dense breasts had a significantly higher uptake of FDG than nondense breasts, a lesion with a SUV of 2.5 or higher should still be easily delineated from the surrounding tissue far better if consider change over time between the early and delayed phases, indicating that the accuracy of FDG-PET in the diagnosis of breast malignancies should not be significantly affected in patients with dense breasts. However, with the significantly higher SUV in normal dense breast parenchyma, it remains to be seen if higher background FDG uptake in patients with dense breast has lower sensitivity in day-to-day reporting of FDG-PET studies in breast cancer. This increased background activity in dense breast may affect visual interpretation of the reader; however, it will not affect the quantitative (SUV) interpretation (kumar). Therefore, we feel that normal breast tissue physiological uptake in the delayed phase imaging would decrease and malignant tissue pathological FDG uptake would increase then tumor to back ground ratio would be higher and this certainly lead 
far better discrimination of tumor lesions.

The association between age and mammographic breast density has been well documented in the literature, with younger women tending to have denser breasts than older women [27-30]. Based on this, one may expect to see lower SUVs in normal breast tissue with increasing age. Our results show significant difference in maximum and average SUVs of breast parenchyma and nipple in patients of different age groups, which is different from the results reported in other studies $[19,26]$. This could explained by the sample number and the different ethnic groups in each study. Furthermore, there were trends of decreasing SUVs with increasing age, which might become significant with larger sample size of patients with different age groups [26].

Of the 284 involved women in this study, 142 were premenopausal, 142 were postmenopausal. Breasts of premenopausal women had a higher SUV than breasts of postmenopausal women. We find significant impact of menopausal status on SUVs, as there is significant difference noted among premenopausal and postmenopausal patients. This finding is similar to the results of Vranjesevic et al. [19], which showed significant effect of hormonal status on SUV uptake. They demonstrated that postmenopausal women receiving hormonal replacement therapy (HRT) had peak and average SUVs similar to those of premenopausal women, whereas the SUVs of postmenopausal women not receiving HRT were significantly lower.

\section{Conclusion}

In summary, this prospective study performed with modern PET/CT scanner demonstrates that, there was a significant difference in SUVs between the dense and nondense normal breast. Menopausal status and age do also have significantly affect the uptake of FDG. The present study suggests that dual time point FDG-PET/CT imaging with SUV change over time would be useful for the assessment of primary breast cancer as it improves the discrimination between physiological and pathological FDG uptake where in the delayed phase imaging the physiological uptake decreases and the pathological uptake increases. This could have good impact and superior sensitivity for the detection of small cancers and cancers in dense breast tissue or breast cancer with minimal FDG uptake.

\section{Acknowledgments}

The author gratefully acknowledges Professor Koji Murakami, Division of Nuclear Medicine, Radiology Department, Keio University, JAPAN, for his valuable assistance. Furthermore, enormous thanks to the secretarial staff at our institution, PET radiochemists for the production of radiopharmaceuticals and PET technicians for skillful acquisition of images.

\section{References}

[1] Jemal A, Murray T, Samuels A, Ghafoor A, Ward E, Thun MJ. Cancer statistics. CA Cancer J Clin. 2003;53:5-26.

[2] Scheidhauer K, Walter C, Seemann MD. FDG PET and other imaging modalities in the primary diagnosis of suspicious breast lesions. Eur J Nucl Med Mol Imaging. 2004;31(suppl 1):S70-S79.

[3] Rosenberg RD, Hunt WC, Williamson MR, Gilliland FD, Wiest PW, Kelsey CA, et al. Effects of age, breast density, ethnicity, and estrogen replacement therapy on screening mammographic sensitivity and cancer stage at diagnosis: review of 183,134 screening mammograms in Albuquerque, New Mexico. Radiology. 1998;209:511-518

[4] Baines CJ, Miller AB, Wall C, McFarlane DV, Simor IS, Jong R, et al. Sensitivity and specificity of first screen mammography in the Canadian National Breast Screening Study: a preliminary report from five centers. Radiology. 1986;160:295-298

[5] Fletcher SW, Black W, Harris R, Rimer BK, Shapiro S. Report of the International Workshop on Screening for Breast Cancer. J Natl Cancer Inst. 1993; 85:1644-1656

[6] Tabar L, Fagerberg G, Chen HH, Duffy SW, Smart CR, Gad A, et al. Efficacy of breast cancer screening by age. New results from the Swedish Two-County Trial. Cancer. $1995 ; 75: 2507-2517$

[7] Frisell J, Klund G, Hellstrom L. Randomized study of mammography screening: preliminary report on mortality in the Stockholm trial. Breast Cancer Res Treat. 1991;18:49-56

[8] Boyd NF, Lockwood GA, Martin LJ, Byng JW, Yaffe MJ, Tritchler DL. Mammographic density as a marker of susceptibility to breast cancer: a hypothesis. IARC Sci Publ. 2001;154:163-169.

[9] Kolb TM, Lichy J, Newhouse JH. Comparison of the performance of screening mammography, physical examination, and breast US and evaluation of factors that influence them: an analysis of 27,825 patient evaluations. Radiology. 2002;225:165-175

[10] Mandelson MT, Oestreicher N, Porter PL, et al. Breast density as a predictor of mammographic detection: comparison of interval- and screen-detected cancers. J Natl Cancer Inst. 2000;92:1081-1087.

[11] Foxcroft LM, Evans EB, Joshua HK, Hirst C. Breast cancers invisible on mammography. Aust N Z J Surg. 2000;70:162167.

[12] Salvatore M, Del Vecchio S. Dynamic imaging: scintimammography. Eur J Radiol. 1998;27 Suppl 2:S259264

[13] Lehman CD, White E, Peacock S, Drucker MJ, Urban N. Effect of age and breast density on screening mammograms with false positive findings. AJR. 1999; 173:1651-1655

[14] Nieweg OE, Kim EE, Wong WH. Positron emission tomographywith fluorine-18-deoxyglucose in the detection and staging of breast cancer. Cancer. 1993;71:3920-3925 
[15] Hoh CK, Schiepers C. 18-FDG imaging in breast cancer. Semin Nucl Med. 1999;29:49-56

[16] Adler LP, Crowe JP, Al-Kaisi NK, Sunshine JL. Evaluation of breast masses and axillary lymph nodes with [F-18]2deoxy-2-fluoro-D-glucose PET. Radiology. 1993; 187:743750

[17] Avril N, Dose J, Janicke F, Bense S, Ziegler S, Laubenbacher C, et al. Metabolic characterization of breast tumors with positron emission tomography using F-18 fluorodeoxyglucose. J Clin Oncol. 1996;14:1848-1857

[18] Alavi A, Kung JW, Zhuang H. Implications of PET based molecular imaging on the current and future practice of medicine. Semin Nucl Med. 2004;34:56-69

[19] Vranjesevic D, Schiepers C, Silverman DH, Quon A, Villalpando J, Dahlbom M, et al. Relationship between 18FFDG uptake and breast density in women with normal breast tissue. J Nucl Med. 2003;44:1238-1242

[20] Kumar R, Schnall MD, Alavi A. 18F-FDG uptake and breast density in women with normal breast tissue. J Nucl Med. 2004; $45: 1423-1424$

[21] Zytoon AA, Murakami K, El-Kholy MR, El-Shorbagy E. Dual time point FDG-PET/CT imaging ... Potential tool for diagnosis of breast cancer. Clin Radiol. 2008; 63(11):121327.

[22] Pisano ED, Yaffe MJ () Digital mammography. Radiology 2005;234: 353-362
[23] Kumar R, Alavi A. Fluorodeoxyglucose-PET in the management of breast cancer. Radiol Clin North Am 2004;42:1113-1122

[24] Zytoon AA, Murakami K, El-Kholy MR, El-Shorbagy E, Ebied O. Breast cancer with low FDG uptake: characterization by means of dual-time point FDG-PET/CT. Eur J Radiol. 2009;70(3):530-8.

[25] Grove JS, Goodman MJ, Gilbert FI Jr, Mi MP. Factors associated with mammographic pattern. $\mathrm{Br} \mathrm{J}$ Radiol. 1985;58:21-25.

[26] Kumar R, Chauhan A, Zhuang H, Chandra P, Schnall M, Alavi A. Standardized uptake values of normal breast tissue with 2-deoxy-2-[F-18]fluoro-D: -glucose positron emission tomography: variations with age, breast density, and menopausal status. Mol Imaging Biol. 2006;8(6):355-62.

[27] Ciatto S, Zappa MA. A prospective study of the value of mammographic patterns as indicators of breast cancer risk in a screening experience. Eur J Radiol 1993;17:122-125

[28] Flook D, Gilhome RW, Harman J, Gravelle IH, Webster DJ. Changes in Wolfe mammographic patterns with aging. Br J Radiol 1987;60:455-456

[29] Wolfe JN. Breast parenchymal patterns and their changes with age. Radiology 1976;121:545-552

[30] Kerlikowske K, Grady D, Barclay J, Sickles EA, Ernster V. Effect of age, breast density, and family history on the sensitivity of first screening mammography. JAMA 1996;276:33-38 